\title{
A Contribuição Francesa ao Ensino e à Pesquisa em Geofísica no Estado da Bahia
}

French contribution to the teaching and geophysics' research in Bahia State

\author{
Aurino Ribeiro Filho, Dionicarlos Soares de Vasconcelos e Olival Freire Jr. \\ Instituto de Física da Universidade Federal da Bahia \\ Campus de Ondina, Salvador, BA, 40.210-340
}

Recebido em 20 de fevereiro, 2003. Aceito em 16 de abril, 2003.

\begin{abstract}
O desenvolvimento de um polo de ensino pós-graduado e de pesquisa em Geofísica, no Instituto de Física da UFBA, nos anos sessenta, do século XX, teve no seu início uma forte cooperação francesa, a qual é aqui resgatada com a finalidade de narrar a sua história e discutir algumas implicações.

The development of a graduate school and research in Geophysics, at the Institute of Physics - UFBA, in Brazil, in the 1960s, started with a strong cooperation of French researchers, which we recover now in order to account its history and to discuss some implications.
\end{abstract}

\section{Introdução}

Até o final dos anos cinqüenta, do século $\mathrm{XX}$, a Universidade Federal da Bahia (UFBA) limitava-se a praticar, quase que exclusivamente, o ensino de graduação para os cursos tradicionais, sem apresentar uma maior vinculação com a manutenção de projetos de pesquisa e de pósgraduação, salvo algumas poucas exceções em que algumas investigações eram empreendidas na Faculdade de Medicina e na Faculdade de Filosofia, Ciências e Letras (FFCL). Este quadro se modificaria radicalmente, a partir do início dos anos sessenta, graças ao empenho de Edgard Rêgo Santos[1], fundador da Universidade da Bahia (UFBA a partir de 1966) em 02.07.1946 que, a partir de um projeto de modernização da Universidade, a elevaria ao patamar de o mais moderno centro universitário do País, àquela época. Foi reitor por um período de dezesseis anos, e naqueles anos implementou o citado projeto no campo das artes e das ciências básicas, além de ter criado o Laboratório de Geomorfologia, liderado pelo geógrafo Milton Santos. No mesmo período surgiram o Instituto de Cultura Hispânica, a Casa da França, o Laboratório de Fonética dirigido por Nelson Rossi e o Centro de Estudos Afro-Orientais (CEAO) sob a direção do pensador português George Agostinho Baptista da Silva. A Universidade da Bahia foi pioneira ao criar os cursos superiores de Dança, Teatro e Música, e com isso conseguiu atrair para os seus quadros muitos artistas e intelectuais, do Brasil e do exterior, influenciando todo o movimento cultural baiano, nos anos sessenta, do século XX, período às vezes denominado de "renascença baiana", e que propiciou, dentre outros eventos culturais, o advento do Ciclo Baiano de Cinema, germe do movimento cinema- tográfico - Cinema Novo, através de um grupo de cineastas capitaneado por Glauber Rocha, Roberto Pires, Rex Schindler, Braga Neto e outros. Para o ensino da Dança Contemporânea foram contratados artistas estrangeiros (Janka Rudzka e Rolf Gelewsky). A Escola de Teatro tornou-se uma das mais respeitadas em todo o mundo graças à direção de Eros Martin Gonçalves e a colaboração de outros artistas, dentre os quais a arquiteta Lina Bo Bardi que fundaria os Museus de Arte Sacra e Arte Moderna. Nas palavras do arquiteto e acadêmico Paulo O. de Azevedo, o renascimento baiano surgiu "dentro de um contexto plural onde se interligavam a derrota do nazi-fascismo e as lutas de emancipação colonial, no plano mundial; o término da ditadura de Vargas e a Constituição de 1946, no plano nacional; o levante de jovens intelectuais baianos, o advento do governo democrático de Mangabeira e a criação da Universidade da Bahia, no plano estadual" [2].

$\mathrm{Na}$ área das ciências básicas destacou-se a criação do curso de Geologia. A Escola de Geologia (origem do atual Instituto de Geociências) teve como primeiro diretor e organizador o físico Ramiro de Porto Alegre Muniz, que iniciou os seus estudos de graduação em 1949 na Faculdade Nacional de Filosofia da Universidade do Brasil. Em 1952, transferiu-se para a Universidade de Berkeley (USA) onde graduou-se e obteve o Mestrado. Ex-funcionário do Banco do Brasil foi apresentado pelo economista Rômulo de Almeida ao reitor Edgard Santos, que o convidaria a exercer o cargo de professor do Departamento de Física da FFCL. Em conjunto com outros docentes participou da criação do Instituto de Matemática e Física (IMF) da então Universidade da Bahia transferindo-se, posteriormente, para a Universidade de Brasília, convidado por Darcy Ribeiro. Anos 
mais tarde foi trabalhar no Centro Brasileiro de Pesquisas Físicas (CBPF). Em 1975 retornou ao atual Instituto de Física da UFBA (IFUFBA), como professor visitante, retornando ao CBPF onde tornou-se pesquisador titular e diretor. Aposentado trabalhou no Observatório Nacional, de onde retornou novamente ao CBPF a fim de dirigir o setor de informatização[3].

O interesse pelos estudos geofísicos e geológicos no Estado da Bahia tem origem na constatação de vários fatores geoclimáticos que singularizam bastante esta região do globo terrestre. A faixa de terreno sedimentar que a atravessa de norte a sul implicou no desenvolvimento da prospecção de jazidas petrolíferas. A observação de clima árido no interior e de clima úmido nas suas regiões costeiras, bem como a presença de enormes extensões de terrenos cristalinos, alguns com forte mineralização e outros, ao contrário, apresentando-se com uma composição muito mais homogênea eram características que sugeriam a criação da citada Escola de Geologia. Segundo Bautista Vidal [4] além dos mencionados aspectos de geologia e da meteorologia, a presença de corais fixos sobre um embasamento estável era um indicativo de que a costa do Brasil servia, por excelência, para o estudo das transgressões marinhas eustáticas do Quaternário, enquanto que o surgimento de concreções nos terrenos calcáreos do interior possibilitaria completar a paleoclimatologia do quaternário recente, ao estudar-se as oscilações térmicas dos últimos milênios nas regiões tropicais, visto que, àquele período, tais estudos já tinham sido implementados nas regiões temperadas boreais.

A presença da Empresa Brasileira de Petróleo S.A. - PETROBRÁS no Estado da Bahia e o forte impacto causado pela indústria petrolífera, no desenvolvimento econômico do País, influenciaram os novos dirigentes da então Universidade da Bahia e alguns jovens físicos, dentre eles o engenheiro civil e físico José Walter Bautista Vidal [5], baiano com pós-graduação no Centro Brasileiro de Pesquisas Físicas (CBPF) e na Universidade de Stanford (USA) que, em conjunto com outros jovens pesquisadores, apresentaria à Universidade, um projeto de criação de um Curso de Especialização em Geofísica. Bautista Vidal enfatiza em seu depoimento que: "Logo que assumi a chefia do Departamento de Física fui a São Paulo e consegui convencer doze jovens físicos para virem para a Bahia. O campo da pesquisa em Geofísica era o grande impulsionador. Os primeiros recursos vieram da Superintendência de Desenvolvimento do Nordeste (SUDENE), onde havia um clima muito favorável ao desenvolvimento das ciências. Na época encarregaram-me da elaboração de um Plano de Desenvolvimento de Ensino e da Pesquisa em Física no Nordeste. Deste Plano surgiram os recursos que permitiram cobrir, parcialmente, a contratação do Grupo de São Paulo, entre os quais se encontravam Humberto Siqueiros Tanure, JeanMarie Flexor, Antônio Expedito Azevedo, Carlos Borba e outros que não resistiram à "fase heróica" do Instituto, ou seguiram outros rumos". Em 1964, iniciou-se um intenso programa de formação de geofísicos - os primeiros da mencionada empresa. Por iniciativa do grupo do Departamento de Física, do então Instituto de Matemática e Física (IMF) da Universidade da Bahia, em convênio com o Centro de Aperfeiçoamento de Pesquisa de Petróleo (CENAP) - PETROBRÁS, foi instalado o citado curso de pós-graduação latu-sensu, com duração de dois anos. Tal curso permitiu a formação de aproximadamente sessenta geofísicos, nas diversas especialidades e que tiveram, em grande parte, a responsabilidade pelos estudos geofísicos das pesquisas na plataforma continental. Graças ao sucesso deste curso foi estabelecido um convênio com a United Nations Educational, Scientific and Cultural Organization (UNESCO) que propiciou a vinda do exterior de doutores em Geofísica que permaneceram não só como visitantes por curtos períodos, mas, também, por vários anos, lecionando e realizando pesquisas, a exemplo de G. Adler, L. P. Geldart, Claude Lalou e Jacques Labeyrie.

Esta foi a gênese do atual Programa de Pesquisa e PósGraduação em Geofísica e Geologia (PPPG), centro de referência no País, o qual foi instalado, no seu início, no Instituto de Física em cooperação com Instituto de Geociências (antiga Escola de Geologia), recém-fundados, em consequiência da implantação da reforma universitária, em 1968. Em setembro daquele ano o grupo de pesquisadores em Geofísica convidaria Carlos Alberto Dias[6], doutor em Geofísica pela Universidade de Berkeley (USA), paraense de origem e que permaneceria por muitos anos na Bahia ajudando a consolidar, em definitivo, o grupo, a partir da implementação de novas linhas de pesquisas em Geofísica Pura e Aplicada, Sedimentologia e Geologia Econômica. Ele redirecionou certas linhas de pesquisa em Geofísica Pura e Aplicada e criou o PPPG, que se tornaria um centro de referência internacional, conseguindo atrair pesquisadores importantes das mais diferentes instituições européias, americanas e asiáticas, graças aos convênios com diferentes agências de fomento e a PETROBRÁS. Por conseguinte, um grande número de estudantes foi absorvido nos cursos de Mestrado e Doutorado em Geofísica, ambos detentores, por muitos anos, de excelente conceito na comunidade brasileira. Em setembro de 1974 (16 a 20) ele presidiu o comitê organizador da I - Reunião Latino-Americana de Geofísica, em Salvador - Ba, com o patrocínio do Centro LatinoAmericano de Física (CLAF), UFBA, UNESCO, Conselho Nacional de Desenvolvimento Científico e Tecnológico $(\mathrm{CNPq})$, Fundação de Amparo à Pesquisa do Estado de São Paulo (FAPESP) e Academia Brasileira de Ciências. Importantes pesquisadores da cena geofísica internacional estiveram presentes a exemplo de J Verhoogen (Universidade da Califórnia), C. Lomnitz (Universidade Autônoma do México), A. Forti (UNESCO) e muitos outros.

O apoio recebido pelo PPPG, tanto da PETROBRÁS quanto das agências de fomento à pesquisa, bem como o êxito desse programa, não podem ser compreendidos fora do contexto da política brasileira de ciência e tecnologia do período. Simon Schwartzman, em seu trabalho "Um espaço para a ciência - A Formação da Comunidade Científica no Brasil - denominou aquela política de "O Grande Salto à Frente", dedicando-lhe todo um capítulo. Segundo esse au- 
tor, essa política decorreu de "uma convergência entre os intelectuais e cientistas da esquerda e os militares da direita", a qual "foi proporcionada pelo nacionalismo e pela crença compartida nos poderes da ciência e da tecnologia;"“ assinalando, em seguida, que "tal coexistência não foi pacífica", e que "muitos intelectuais perderam seus cargos acadêmicos e se viram forçados ao exílio". Dentre as diversas expressões daquela política, ele identifica a criação do atual Instituto Alberto Luiz Coimbra de Pós-Graduação e Pesquisa de Engenharia (COPPE), no Rio de Janeiro, o apoio massivo à Universidade Estadual de Campinas, e a criação de novos programas de pós-graduação [7]. Escrever a história da introdução do ensino e da pesquisa em Geofísica, no Instituto de Física da UFBA, contribuirá para uma melhor compreensão daquela política. Nosso objetivo neste texto é, contudo, bem mais limitado, e se prenderá a evidenciar aspectos da sua origem que foram obscurecidos pela sua "história oficial".

Em linhas gerais o roteiro histórico anteriormente apresentado é o mais conhecido [6] o qual, apesar de verdadeiro, não é completo. Em realidade, antes de atingir a sua pujança nos anos setenta e oitenta, do século passado, dentre os primeiros bandeirantes, nos anos sessenta, como afirmado antes, estavam aqueles participantes do grupo de jovens físicos baianos graduados na UFBA, na Universidade de São Paulo (USP) e na antiga Universidade do Brasil. Eles não tinham uma grande experiência científica, porém apresentavam um grande interesse em introduzir na jovem universidade um trabalho sério de ensino e pesquisa no campo da Geofísica Nuclear e Aplicada.

Bautista Vidal [5] lembra os esforços que culminaram no estabelecimento de um intenso programa de pesquisa e treinamento com o Centre des Faibles Radioactivités (CFR), em Gif-sur-Yvette, França. O interesse pelo campo da Geofísica tinha por inspiração além da PETROBRÁS, o enorme potencial geológico do Estado da Bahia, com a sua reconhecida riqueza mineral. O fato é que a criação do Laboratório de Fracas Radioatividades (LFR), o hoje denominado Laboratório de Física Nuclear Aplicada (LFNA) do Instituto de Física da UFBA, foi inspirada no seu congênere francês (Laboratoire de Faibles Radioactivités) situado na mencionada cidade francesa. Com referência à citada instituição francesa, além do apoio acadêmico foi também o centro de inspiração e de treinamento de muitos dos jovens docentes da Bahia, cujos depoimentos e críticas fornecem uma idéia geral sobre algumas questões que pontificaram a introdução da pesquisa geofísica na Bahia. Antônio Expedito de Azevedo (espectroscopia isotópica) [8], relembrando a sua fase inicial afirmou que "A possibilidade da vinda para a Bahia surgiu em 1964, após a visita à USP de Bautista Vidal (...). Em dezembro de 1964 vim, com os colegas Jean Flexor, Humberto Tanure, Mauro Catani e José Galvão, para uma visita à Bahia e discussão de um programa a ser desenvolvido na Universidade. Daquele grupo, Jean, Humberto e Mauro se incorporaram à UFBA logo no início de 1965. Eu só resolvi me transferir em outubro de 1965. Galvão preferiu continuar sulista"... "O intercâmbio com o Centre des Faibles
Radioactivités, de Gif-sur-Yvette, França, permitiu, a curto prazo, criar as condições para a implantação do LFNA (LFR). Essa implantação foi viabilizada também pela reforma e ampliação da UFBA que, a partir de 1969, implantou a nova estrutura de Institutos Básicos com um grande investimento em instalações e equipamentos"..."Minha área de trabalho inicial na Geofísica Nuclear foi a de isótopos estáveis, depois que aprendi com Jean Claude Duplessey, durante 1968, em Gif-sur-Yvette, a usar um espectrômetro de massa para a medida de razões isotópicas de carbono e oxigênio em carbonato ..."; Lycia Maria Nordemann (espectrometria alfa) [9] graduou-se em Física pela FFCL, da UFBA, e especializou-se em Gif-sur-Yvette, França, sob a orientação de Claude Lalou, na área de espectometria alfa. Retornou ao IFUFBA onde exerceu atividades docentes e de pesquisa no LFNA, onde mais tarde concluiu uma série de trabalhos experimentais para a sua tese de Doctorat d'État en Sciences Naturelles; Humberto Siqueiros Rodrigues Tanure (aerossóis atmosféricos e traços de fissão), que foi diretor do IFUFBA e observador privilegiado de todo aquele processo de implantação do projeto de Geofísica, em depoimento, afirmou [10]: "O meu trabalho na França, nos anos 69/70, foi na área dos aerossóis naturais, sobretudo os de origem marinha. O Laboratório onde estive, tinha feito nas décadas de cinquenta e sessenta inúmeros trabalhos sobre aerossóis radioativos, oriundos das explosões nucleares na atmosfera, que permitiram o estudo da estrutura da atmosfera em nível global. Com o final desses testes a atenção se voltou para os aerossóis naturais, com o intuito de se estudar o ciclo geoquímico de elementos"..."Os atritos entre o IFUFBA $e$ o PPPG, que durante a minha gestão (diretor do IF) assumiram por vezes grandes dimensões, se originaram basicamente da desproporção entre as dimensões efetivas daquele programa (PPPG) e seu precário estatuto legal na Universidade. Também os contornos entre as entidades eram muito mal definidos, o que dava origem a in úmeros conflitos de autoridade"..."Hoje eu acho que a Geofísica Nuclear foi uma escolha infeliz, fruto da nossa imaturidade no momento de se tomar as grandes decisões. De fato, os problemas abordados nesse campo são quase sempre multidisciplinares, $e$ a Física entra aí em pequeníssima proporção. Em particular, a experiência de todos estes anos aqui mostrou que pessoas com formação em Física, são as menos indicadas para estudar problemas ambientais, como florestas, desertos, poluição..."; Jean-Marie Flexor (geocronologia nuclear por carbono - 14) [11] bacharel em Física pela USP, em 1965 incorporou-se ao citado grupo de jovens físicos liderado por Bautista Vidal que lutou pela implantação de uma "nova" Física voltada para os interesses nacionais. Especializouse em Gif-sur-Yvette, França, em técnicas de datação pelo método de isótopos de urânio e tório por espectrometria alfa. Trabalhou em técnicas de detecção nuclear no Service d'Electronique Physique do Centro de Estudos Nucleares de Saclay. Com a criação do LFNA ele tornou-se o seu líder científico orientando inúmeras dissertações de Mestrado e escreveu a sua tese doutoral, defendida na USP. A partir de 1979 , o seu trabalho de pesquisa expandiu-se para ou- 
tras áreas das geociências, interagindo fortemente com sedimentologistas da UFBA e da USP no projeto de "estudos sobre o Quaternário", cujos resultados formam hoje um enorme conjunto bibliográfico. Convidado para dirigir o setor de Geofísica do Observatório Nacional, no Rio de Janeiro, ele transferiu-se para aquela instituição, interagindo também com pesquisadores uspianos; Roberto Max de Argollo (espectrometria gama) [12] interrompeu o seu curso de Engenharia na Escola Politécnica da UFBA, e concluiu o bacharelado em Física no Rio de Janeiro, especializando-se, posteriormente, em espectrometria gama no CBPF. Ao retornar à UFBA lecionou na Escola Politécnica, transferindose mais tarde para o IMF participando do grupo fundador de Geofísica e do LFNA. Trabalhou, inicialmente, nesse laboratório com pesquisadores de Gif-sur-Yvette, França, a exemplo de Daniel Nordemann e Patrick Buat-Ménard, no período em que pesquisadores de outras instituições de Mônaco (Jean-Louis Rapaire), da antiga República Tcheca (Emanuel Klier) e da Polônia (Zbigniew Baran) trabalhavam no LFNA. A partir de 1972 foram concluídas as primeiras dissertações de Mestrado em Geofísica Nuclear, as quais foram orientadas pelos professores visitantes tendo a colaboração dos jovens docentes (com especialização) do Instituto de Física.

A influência acadêmica francesa na própria UFBA era intensa, àquela época, graças à fundação da Casa da França, no próprio campus universitário do Canela [13]. Por conseguinte, muitos jovens pesquisadores franceses foram trazidos pelo Serviço de Cooperação, para distintas áreas da universidade, em particular, para a Curso de Geografia. Foi instalado, por tal influência, o Laboratório de Geomorfologia onde um programa de Geografia Humana intensificouse graças, também, à liderança do saudoso geógrafo baiano, de renome internacional, Milton Santos, que doutorou-se na França, e em decorrência de perseguição política, pelo regime militar, sairia do Brasil e desenvolveria um intenso trabalho de pesquisa, em vários continentes, que proporcionoulhe, mais tarde, o mais importante prêmio internacional de Geografia - o Vautrin Lud. Vale ressaltar que o projeto de pesquisa em Física dos aerossóis atmosféricos desenvolvido no LFR beneficiou-se bastante dos trabalhos de campo em Geografia e Geomorfologia desenvolvidos na Bacia do Rio Paraguaçu (Bahia), uma das regiões de interesse de diferentes projetos de pesquisas implementados por diferentes grupos de pesquisadores da UFBA, àquela época.

\section{O "LFR" e a geofísica nuclear}

Em 1967, o Departamento de Física do então IMF, encaminhou à reitoria da UFBA o projeto de desenvolvimento de um "Laboratório de Fracas Radioatividades" (LFR). Tal projeto originou-se de um relatório elaborado, após uma viagem de estudos à França, em 1966, realizada por Bautista Vidal, chefe do mencionado departamento; e uma outra, em início de 1967 ao Brasil (Salvador - Bahia) pelos pesquisadores franceses Dra. Claude Lalou, chefe de pesquisas no CFR, e Professor Jacques Labeyrie, diretor do "Service d'Electronique Physique" - Centro de Estudos Nucleares de Saclay em Gif-sur-Yvette.

O citado relatório resumia as discussões entre os mencionados três pesquisadores e demais colaboradores, sobre as linhas de pesquisa a serem desenvolvidas pelo programa nos próximos anos na Universidade Federal da Bahia. A opção para que as pesquisas devessem ser no ramo da Geofísica fundamentou-se em alguns postulados que, em síntese, determinavam que: “(i) A pesquisa deveria ser ligada aos interesses nacionais, tendo por motivação as vantagens, no domínio científico, da situação geográfica e geofísica e da estrutura geológica desta região do globo terrestre; (ii) Os trabalhos deveriam ser desenvolvidos numa área da ciência em franca produção, utilizando-se métodos modernos e com isso despertar o interesse de jovens brasileiros incentivandoos a interagir com pesquisadores nacionais e de outros países; (iii) A utilização de uma instrumentação moderna e a conseqüente criação de um núcleo de apoio técnicoeletrônico local, a fim de minimizar a dependência externa e um melhor aproveitamento de tais equipamentos; (iv) A ênfase em trabalhos de pesquisa fundamental que pudesse atrair os melhores pesquisadores e criar os desenvolvimentos originais que orientariam as futuras etapas e, em paralelo, voltar-se-iam para as aplicações práticas, a fim de contribuir para o desenvolvimento tecnológico e industrial imediato". A partir de tais premissas o jovem grupo de pesquisa deveria procurar desenvolver na UFBA um ensino pósgraduado (e de graduação) de alto nível que viesse assegurar o desenvolvimento ulterior das pesquisas [5].

O programa de ensino e pesquisas foi baseado na utilização de instalações, no LFR, de: (i) datação pelo $C^{14}$; (ii) espectrometria alfa a semicondutores; (iii) espectrometria gama por cristais $N a I$; (iv) datação pelo método de potássio-argônio - por espectrometria de massa; e (v) extensão ulterior eventual para a determinação das relações isotópicas $O^{18} / O^{16}$. Ao lado de tal infraestrutura experimental havia outras proposições para o futuro, a exemplo de uma instalação de estudos sísmicos ; uma instalação temporária de lançamento de balões; instalações para preparação de estudos magnetométricos terrestres e oceânicos e de dragagens em águas profundas e a medidas sísmicas oceânicas. Apesar de não terem sido executadas naquele início, algumas dessas últimas instalações foram implementadas pelo atual Programa de Pesquisa em Geofísica e Geologia da UFBA. Com referência aos ítens (i) a (v) citados eles eram praticamente idênticos aos já existentes no CFR do Centre National de la Recherche Scientifique (CNRS) em Gif-sur-Yvette - França. Tal determinação motivou que a sua montagem bem como o seu funcionamento implicasse na necessidade de estágios, de jovens pesquisadores baianos, naquele centro de pesquisas.

No período 1972 a 1982 vários trabalhos foram publicados em revistas especializadas de circulação nacional e internacional, além de inúmeras comunicações, bem como várias dissertações que tiveram por base estudos geofísicos nas diferentes regiões do Estado da Bahia. Alguns docen- 
tes trabalharam em projetos de doutoramento, defendendo tese na Universidade de São Paulo e em outras instituições no exterior. Naquele período, as pesquisas desenvolvidas enquadravam-se em diferentes linhas específicas: Hidrologia Isotópica, Ciclo do Carbono, Paleoclimatologia, Física dos Aerossóis Atmosféricos e Espectrometrias. A primeira apoiava-se na determinação da composição isotópica da água e de elementos nela dissolvidos para o estudo do ciclo hidrológico e da dinâmica de águas subterrâneas na região. Os estudos sobre o Ciclo do Carbono referiam-se à distribuição e intercâmbio do carbono nos reservatórios da atmosfera, biosfera e oceanos e como bem relembra Azevedo (1968) [14]: “Um dos métodos de datação mais populares de geocronologia é o Carbono - 14. Foi desenvolvido por Libby e posto em base praticável a partir de 1949”. (...) "O Carbono - 14, um isótopo radioativo do Carbono, é formado continuamente na alta atmosfera, devido ao bombardeamento de átomos de nitrogênio por raios cósmicos. Como esse bombardeamento pode ser considerado, em média, como constante no tempo, fica estabelecido um equilíbrio secular na quantidade de Carbono - 14 existente, sendo formada a mesma quantidade que é desintegrada” (...) O Carbono - 14 é largamente usado para datar eventos de menos que 30.000 anos. Com esse método foram modificados muitos conceitos sobre o Pleistoceno recente ...". $\mathrm{Na}$ área da Paleoclimatologia o objetivo era o de utilizar a determinação das razões isotópicas do carbono e oxigênio em amostras de carbonetos, associadas à datação dessas amostras por $C^{14}$ ou pelas séries radioativas de urânio e tório, para o estudo das variações de alguns parâmetros ambientais na região tropical, associadas aos ciclos glaciais do Quaternário. A pesquisa em torno dos aerossóis naturais foi desenvolvida utilizando-se dados obtidos de sete estações meteorológicas, da Bacia do Rio Paraguaçu - Bahia, onde amostras de precipitações atmosféricas foram coletadas durante dois anos. Uma razoável quantidade de amostras foram analisadas por meio das técnicas de espectrometrias gama e alfa.

As primeiras publicações em torno da geocronologia via carbono explicitavam os estudos de evolução da matéria orgânica em solos, apresentados por Flexor et al (1972), Lôbo (1972), Rapaire et al (1973), Flexor et al (1975), Queiroz (1975), Lôbo et al (1974) e Flexor et al (1977) [15].

Com referência ao estudo da paleoclimatologia a experiência do grupo centrou-se no uso de isótopos estáveis de carbono e oxigênio como indicadores de parâmetros do meio ambiente, em geral associado à datação por $C^{14}$. Alguns trabalhos foram publicados nessa área, a exemplo de Duplessey, Lalou et Azevedo (1969), Campos (1976), Pina (1976) e Takahashi e Azevedo (1982) [16].

A análise de precipitações atmosféricas foram reportadas por Tanure et al (1973 e 1974), Buat-Menard et al (1974), Ribeiro Filho (1975), Ribeiro Filho e Tanure (1978) [17]. Posteriormente, o uso de dados químicos e isotópicos de águas de precipitações foi estendido com a incorporação desse tipo de análise em águas de superfície e subterrâneas visando, sobretudo, determinar a dinâmica de circulação da água subterrânea em alguns aqüíferos do Estado da Bahia. Alguns trabalhos discutiram tais resultados: Gomes (1978), Cabral (1978), Siqueira (1978) e Gomes e Cabral (1981) [18].

Diferentes trabalhos e dissertações de Mestrado explicitam as pesquisas em espectrometria gama, espectrometria alfa, radiocristalografia do quartzo, termoluminescência e fluxo térmico e magnetismo: Carvalho (1972), Nordemann e Sakai (1973), Shignolfii e Sakai (1974), D’Aguiar Neto (1974), Azevedo (1975), Pregnolatto (1975), Ferreira (1973), Ferreira (1973), Jucá (1973), Ferreira e Nordemann (1976), Suguio et al (1976), Sakai e Shignolfi (1977), Nordemann (1977), Sakai (1977), Pinto (1977), Novaes (1978), Carvalho (1981) e Jucá (1981) [19].

\section{Conclusão}

O interesse pela melhoria do ensino de Física, nos diferentes níveis, e o desenvolvimento da pesquisa no Instituto de Matemática e Física da UFBA, foi uma meta primordial do grupo de físicos e matemáticos que se instalou no início dos anos 60 nessa instituição. Vários daqueles pesquisadores, dentre os quais destacaram-se: Ramiro de Porto Alegre Muniz, Omar Catunda, Rubens Gouveia Lintz, José Walter Bautista Vidal, Waldez Alves da Cunha, Luiz Felipe Perret Serpa, Arlete Cerqueira Lima, Martha Maria de Souza Dantas, Álvaro da Silva Ramos, Benedito Leopoldo Pepe, Antônio Expedito Gomes de Azevedo, Roberto Max de Argollo, Jean-Marie Flexor, Humberto Siqueiros Rodrigues Tanure, tiveram uma participação decisiva na reforma universitária de 1968, cuja culminância foi a instalação da pesquisa e pós-graduação como partes indissociáveis (junto com o ensino de graduação e a extensão) da vida departamental e do trabalho universitário. A fundação do antigo IMF da Universidade da Bahia, no início dos anos sessenta, foi um marco decisivo na implementação do ensino, em bases modernas, das disciplinas ligadas aos cursos de Matemática e Física, para alunos dos cursos de Engenharia, bem como aqueles que já eram estudantes de Geologia, Bacharelado e Licenciatura em Física e em Matemática, respectivamente [20]. A partir de então, o citado grupo de pesquisadores trabalhou para que houvesse o desmembramento do antigo IMF, nos atuais IFUFBA e Instituto de Matemática da Universidade Federal da Bahia (IMUFBA). Os vários institutos da nova UFBA (dentre os quais os dois citados e o de Geociências) foram definidos como Unidades de Ensino e Pesquisa Básicos, através do decreto 62241, de oito de fevereiro de 1968, que estruturou a Universidade Federal da Bahia.

A partir, portanto, de 1968 expandiu-se o jovem grupo de ensino e pesquisa em Geofísica Nuclear - o que conduziu à instalação do LFR (atual LFNA) - graças ao apoio do grupo de geofísicos franceses sediado em Gif-sur-Yvette (França). O trabalho empreendido no período (1968-1982) pelo grupo baiano foi marcante, devido ao razoável número de pesquisadores visitantes, alunos de pós-graduação e dos 
recursos financeiros advindos de diferentes agências e organismos internacionais tais como UNESCO, Banco Interamericano de Desenvolvimento (BID).... Todo aquele despertar para o trabalho de pesquisa começou a consolidar-se com o surgimento das citadas publicações internas, dissertações de mestrado, teses doutorais, comunicações e artigos em jornais de circulação internacional, em torno dos projetos de pesquisa em Física dos aerosóis atmosféricos, Geocronologia, Evolução da Matéria Orgânica em Solos Tropicais, Radiocristalografia, Espectrometrias Alfa, Gama, de Isótopos Estáveis e de Absorção Atômica, além de estudos do Ciclo do Carbono 14, Hidrologia Isotópica, Paleoclimatologia, Geotermia, Dinâmica Atmosférica e Geofísica Teórica. No citado período consolidou-se a cooperação com o Instituto de Geociências da UFBA e, por conseqüência, expandiu-se a Geofísica para as áreas Aplicada e Teórica, surgindo novas contribuições científicas e, por conseguinte, o aparecimento de outros tópicos interdisciplinares [21].

O impacto do trabalho deste jovem grupo de ensino e pesquisa em Geofísica, nos anos sessenta do século XX, ensejaria Leite Lopes [22] a escrever: "Na Universidade da Bahia, Bautista Vidal organiza um Instituto de Física voltado para os estudos geofísicos de importância fundamental para o desenvolvimento do País"

Alguns momentos de crise atingiriam mais tarde o PPPG, além do surgimento de discordâncias entre jovens pesquisadores que dificultaram em diferentes etapas o relacionamento e a tranqüilidade do ambiente de trabalho. A liderança do setor de Geofísica Aplicada instalado no Instituto de Geociências se, por um lado, conseguia propiciar a necessária estabilidade ao programa, por outro, em vista de posições filosóficas, de como direcionar a temática geofísica, criaria sérios problemas, principalmente, no LFR, onde se estabelecia uma outra linha de pensamento no grupo. Tais conflitos foram agravados pela precária institucionalidade do programa, característica que era, aliás, comum a várias das iniciativas do período do "Grande Salto para Frente", para retomar a expressão de S. Schwartzman [23]. Conforme sua observação sobre tais questões: "a estratégia adotada pelas agências de ciência e tecnologia consistia em identificar os grupos de pesquisa que elas consideravam bons ou promissores e fornecer-lhes apoio direto, freqüentemente contornando normas estabelecidas para firmar contratos de trabalho, procedimentos contábeis e até mesmo o processo de decisão interno às universidades."

Apesar de superada a crise, jamais o LFR conseguiria retornar àquela fase produtiva dos anos sessenta e setenta. Outros aspectos foram abordados por Ramos [24] que justificou a sua não adesão ao projeto de Geofísica: "Naquilo que a nós pessoalmente diz respeito, o mencionado projeto, pelo menos nas versões iniciais em que era concebido, pareceunos exageradamente ambicioso, tanto no porte quanto na grandiosidade da área científica a que se impunha estender suas ações".

Sobre as dificuldades em se implantar um programa daquelas proporções, levando-se em conta a notória inexistência de um maior reconhecimento de outros setores da
Universidade, bem como o incômodo causado em alguns outros com as facilidades de financiamento e de instalações obtidas pelo grupo de Geofísica, é interessante denotar que, por parte de alguns membros do recém-criado PPPG surgiria, de fato, um interesse em extrapolar os seus domínios (Institutos de Física e de Geociências) para diferentes áreas da UFBA, num projeto multidisciplinar, sem contudo observarem as implicações políticas e logísticas de setores mais conservadores da própria Universidade. Ramos [25] mais uma vez relembra que: "Como decorrência imediata daquela mesma filosofia, resultaria envolvimento unilateral de várias unidades recém-criadas - e ainda não consolidadas - num relacionamento presumivelmente difícil, se não impossível. É que, essa mesma decorrência, acrescida das suas naturais implicações, desenhava um quadro claramente próprio ao surgimento de problemas, que por sua vez, se desdobrariam desde a eclosão de crises de natureza puramente políticas até impasses (até quando insuperáveis?) no plano administrativo-institucional".

Enfim, em todo aquele árduo trabalho inicial do mencionado grupo de Geofísica, na Bahia, a cooperação dos pesquisadores franceses de Gif-sur-Yvette foi importante, pois a partir de então todo um trabalho de ensino e pesquisa foi ampliado, apesar de um notório recesso durante os anos noventa do século passado, com a saída e/ou aposentadoria de alguns de seus pesquisadores mais atuantes e o encerramento da citada cooperação internacional. Houve de fato uma crise e um esvaziamento muito grande no setor da Geofísica Nuclear. Uma das causas foi também o deslocamento de prioridades do atual Centro de Pesquisa e PósGraduação em Geofísica e Geologia. Na atualidade o que se observa é a tentativa de um retorno aos áureos tempos do LFR (ou LFNA), com a volta de antigos pesquisadores e a contratação de outros novos, com o fito de implementar novos projetos em Geofísica Nuclear e Ambiental.

\section{Referências}

[1] A. Ribeiro Filho: "História do Desenvolvimento do Ensino e da Pesquisa em Física na Bahia: Notas Introdutórias“, In: Cadernos de Física da Universidade Estadual de Feira de Santana (UEFS), vol. 1, no 1, pp. 9-24 (1996): O reitor Edgard Santos era médico, professor catedrático da Faculdade de Medicina, ex-Ministro da Educação e progenitor do também futuro reitor Dr. Roberto Filgueiras Santos, ex-Governador da Bahia, ex-Ministro da Educação, ex-Deputado Federal e ex-Presidente do Conselho Nacional de Desenvolvimento Científico e Tecnológico (CNPq) e que concluiu a reforma universitária de 1968 na UFBA.

[2] A. Ribeiro Filho e D.S. de Vasconcelos: A Influência Francesa no Programa de Geofísica da UFBA. Anais do VIII SNHCT. Observatório Nacional. Ministério de Ciência e Tecnologia (MCT), Rio de Janeiro-RJ, pp 14-15 (2001); A. Ribeiro Filho: "Glauber Rocha Revisitado", Ed. UESB/UFBA, $135 \mathrm{pp},(1995)$.

[3] R. de P. A. Muniz, Depoimento. Cadernos do IFUFBA, Salvador-Ba, ano1, $\mathrm{n}^{o} .2$, pp 62-78, maio. (1985). 
[4] J. W. Bautista Vidal, C. Lalou e J. Labeyrie: "Projeto de Desenvolvimento de Um Laboratório de Fracas Radioatividades na Universidade Federal da Bahia". Relatório Científico. IMF. UFBA. Salvador. Bahia, pp 1-20 (1967). In: Jornal Universitário. Vol. II. No 17, p.2 (1968). Vide: J. Labeyrie, In: Anais da Acad. Bras. de Ciências. Brasil. 40, pp 31-37 (1968).

[5] J. W. Bautista Vidal, "Depoimento". Cadernos do IFUFBA, ano 1, vol. 2, no 3, pp 54-86, julho (1985).

[6] C. A. Dias, 1984 - PPPG-UFBa: Instituição de vanguarda em pesquisa e ensino pós-graduado de Geofísica e Geologia no Brasil, Salvador, Ed. PPPG/UFBa, 151 pp, mimeo; O. A. L. de Lima e Y. de A. Ferreira (eds.), PPPG/UFBa, 1991 - Perfil da instituição e desempenho técnico-científico (1984-1991), Salvador, PPPG/UFBa, 259 pp., mimeo: Carlos Alberto Dias foi o primeiro $\mathrm{PhD}$ em Geofísica a vir para a Bahia, a convite do grupo já existente àquela época no Instituto de Física.

[7] S. Schwartzman, - Um Espaço para a Ciência - A Formação da Comunidade Científica no Brasil, Brasília, MCT - CNPq - CEE, 2001, Cap. 9, pp. 276-307. O impacto - na Geofísica - das mudanças ocorridas em torno de 1970 na política brasileira de ciência e tecnologia ainda está, nos parece, por ser estudado. Para a história das Geociências, em períodos anteriores, ver S. F. M Figueiroa - As ciências geológicas no Brasil : uma história social e institucional, 1875-1934, São Paulo: Ed. Hucitec, 1997; F. Azevedo (ed) - As ciências no Brasil, Rio de Janeiro: Ed. da Universidade Federal do Rio de Janeiro (UFRJ), $1994,2^{a}$. ed., especialmente os Capítulos IV-VI, pp. 233-347; e M. G. Ferri e S. Motoyama, (eds) História das Ciências no Brasil, São Paulo: EPU, 1979. Para uma visão de conjunto da formação da comunidade científica brasileira, ver S. Schwartzman, op.cit.

[8] A. E. G. de Azevedo, "Depoimento". Cadernos do IFUFBA, ano 1, no 4, pp 42-50, setembro (1985).

[9] L. M. Moreira et C. Lalou: “Étude Expérimentale du Rapport 237U/238U dans les Eaux Naturelles ayant Transversé Differents Types de Roches". In: Anais da Acad. Bras. de Ciências. Brasil. 44, pp. 13-18 (1972); D. Nordemann e L. M. M. Nordemann: Elementos de Geocronologia Nuclear. Texto didático. Ed. Núcleo de Recursos Didáticos $n^{o}$ LIX. UFBA. Salvador. 52 pp (1972); L. M. M. Nordemann and G. Sieffermann: "Distribution of Uranium in Soil Profiles of Bahia State, Brazil". In: Soil Science. Vol. 127, no 5, 5 pp. (1978); "Étude de la Vitesse d'Alteration des Roches au Moyen de l'Uranium Utilisè comme Traceur Naturel. Applicatión a Deux Basins du Nordest du Brésil". Tese de Doctorat d'État. Université Pierre et Marie Curie, Paris VI. Paris. France. 162 pp. (1977).

[10] H. S. R. Tanure, "Depoimento". Caderno do IFUFBA, ano 1, no 4, pp 31-41, setembro (1985).

[11] J-M. Flexor "Medida da Relação $C^{14} / C^{12}$ : Aplicação à Dinâmica do Carbono no Solo". Tese de doutorado, apresentada ao Instituto de Física da USP. Salvador, Ba., 90 pp (1972).

[12] R. M. de Argollo and J. G. Schilling: "Ge-Si and Ge-Al Fractionation in Hawaiian Volcanic Rocks" In: Geochem. Cosmoch. Acta, Holanda, 42, 6, pp 623-630 (1978); "Simultaneous Determination of Gallium and Germanium in Igneous
Rocks by Neutron Activation" In: Analyt. Chem. Acta, Holanda, 96, 1, pp. 117-123 (1978) e "Ge/Si and Ga/Al Variation along the Reykjanes Ridge and Iceland" In: Nature, UK, 276, 5683, pp. 24-28 (1978) ": R. M. de Argolo escreveu a sua dissertação de MSc in Oceanography : "Ga/Al and $\mathrm{Ge} / \mathrm{Si}$ in Volcanic Rocks", na Universidade de Rhode-Island (USA). A partir de 2000 tornou-se professor titular do Departamento de Geofísica Nuclear, do IFUFBA. É o atual chefe do LFNA e, em 21.02.2001, defendeu a sua tese doutoral, junto ao PPPG : "Cronologias de Sedimentação Recente e de Deposição de Metais Pesados na Bahia de Todos os Santos Usando $\mathrm{Pb}^{210}$ e $\mathrm{Cs}^{137}$,

[13] Para as relações científicas Brasil - França, em um período anterior (1850-1950), ver A. I. Hamburger; M. A. Dantes; M. Paty e P. Petitjean (eds) - A ciência nas relações Brasil França (1850-1950), São Paulo, EDUSP, 1996.

[14] A. E. G. de Azevedo, "Geocronologia na U.F.Ba", Jornal Universitário. Departamento Cultural. UFBA. pp. 4, 30.04 (1968): Antônio Expedito Gomes de Azevedo, ex- Coordenador do IF-UFBA, é PhD pela Universidade de Colúmbia, NY, USA, onde defendeu a tese "Atmospheric Distribution of Carbon Dioxide and its Exchange with the Biosphere and the Oceans", NY, 144 pp (1981). Aposentado, transferiu-se para a Rio de Janeiro, retornando, posteriormente, ao LFNA onde tem orientado alunos de Mestrado e Doutorado em Geofísica:

[15] J-M. Flexor, P. F. S. Lôbo e J-L. Rapaire: "Estudo da Evolução da Matéria Orgânica do Solo Utilizando o C - 14 produzido nos Ensaios Termonucleares Atmosféricos". In: Rev. Bras. Geoc. 2. 4. pp 260-269 (1972); P. F. S. Lôbo: "Utilização do C-14 Atmosférico como Traçador da Matéria Orgânica dos Solos”, dissertação de Mestrado. 63 pp (1972); J-L.Rapaire, P. F. S. Lôbo; J-M Flexor et G. Sieffermann: "Determination du Taux de Carbone et du Rapport Isotopique $\mathrm{C}^{14} / \mathrm{C}^{12}$ d'un Horizon d'Alteration Isovolume Ferralitique de la Région Tropicale Humide de Bahia (Brésil)". In: Boll. Group Français Argiles. France; t. XXV, pp.149-153 (1973); P. F. S. Lôbo, J-M. Flexor; J-L. Rapaire et G. Sieffermann: "Essai de Determination du Temps de Residence des Fraccions de Deux Sols Ferralitiques par l'Utilization du Radiocarbone Naturel et Termonucléaire". In: Cahiers ORSTOM. Sér Pedol. France, 12, 1-2, pp 115-123 (1974); J-M Flexor, J. J de Oliveira; J-L. Rapaire et G. Sieffermann: "La Dégradation des Illites et Montmorillonite dans l'Alios de Podzols Tropicaux Humo-Ferrugineux du Recôncavo Bahiano et du Pará". In: Cahiers ORSTOM. Sér. Pedol. France, t. XIII.1, pp. 41-48 (1975); F. C. de Queiroz: "Distribuição da Radioatividade Natural no Perfil de um Podzol Tropical e suas Implicações Genéticas”, dissertação de Mestrado. 51 pp. (1975). J-M Flexor, et B. Volkoff: "Étude du Rapport $\mathrm{C}^{13} / \mathrm{C}^{12}$ dans les Fractions Organiques du Sol”. In: Compte Rendus de l'Acad. Sc. Paris. France, t. 284, pp. 1655-1657 (1977).

[16] J. C. Duplessey, C. Lalou et A. E. G. Azevedo: "Étude des Conditions de Concretionnement dans les Grottes au Moyen des Isotopes de l'Oxygene et du Carbone". In: Compte Rendus de l'Acad. Sc. Paris. France, 268, 20, pp. 2327-2330 (1969); H. S. Campos, "Estudo das Variações Isotópicas $\mathrm{C}^{13} / \mathrm{C}^{14}$ e $\mathrm{O}^{18} / \mathrm{O}^{16}$ em Ambientes de Formação de Rochas de Práia na Ilha de Itaparica-Bahia", dissertação de Mestrado. 55 pp, (1976); L. C. Pina: "Estudo do Comportamento dos Elementos C, O, Ca, Mg no Estuário do Rio Paraguaçu - Bahia”, 
dissertação de Mestrado. 52 pp. (1976); T. Takahashi, and A. E. G. Azevedo: "The Oceans as a $\mathrm{CO}_{2}$ Reservoir". In: Reck, $\mathrm{R}$ and $\mathrm{J}$ Hummer (eds): Interpretation of Climate and Photochemical Models, Ozone and Temperature Measurements. American Institute of Physics (AIP). NY. USA, pp. 83-109 (1982).

[17] H. S. R. Tanure, H. Steinberg; G. C. Magalhães e A. C. V de Athayde: "Projeto de Implantação e Manutenção de um Banco de Dados Meteorológicos - Sistema MET”. In: Informativo Meteorológico. DNMET. Brasil. Nov/Dez (1974); H. S. R. Tanure, A. Ribeiro Filho, T. Sakai et P. Buat-Menard: "Concentrations en $\mathrm{Na}, \mathrm{K}, \mathrm{Ca}, \mathrm{Mg}$ dans les Pluies Cotiéres et de l'Interieur de l'État de Bahia (Brésil)". In: Bull de l'Union des Oceanographes de France. Special Issue. C21, (1973); P. Buat-Menard, H. S. R. Tanure, A. Ribeiro Filho, T. Sakai and R. Chesselet: "Sources of $\mathrm{Mg}, \mathrm{K}$ and $\mathrm{Ca}$ Enrichment in Coastal and Inland Precipitations over the Bahia State (Brazil)". In: Journal de Recherc. Atmospheriques. France. VIII, 3-4, pp. 995-997 (1974);A. Ribeiro Filho: "Comportamento do Sódio, Magnésio, Cálcio e Potássio nas Precipitações Atmosféricas na Bahia do Paraguaçu - Bahia ", dissertação de Mestrado, 75 pp, (1975); A. Ribeiro Filho e H. S. R. Tanure: "Estudo das Razões $\mathrm{Mg} / \mathrm{Na}, \mathrm{Ca} / \mathrm{Na}$ e K/Na nas Águas de Chuva da Bacia do Paraguaçu, Brasil”, In: Rev. Bras. Geoc., Brasil, 8, 1, pp. 23-32 (1978).

[18] F. V. M. Gomes: "Aplicações dos Isótopos do Urânio como Traçadores de Água Subterrânea do Bambuí-Bahia”, dissertação de Mestrado, 73 pp, (1978); F. C. F. Cabral: "O Uso dos Isótopos do Carbono no Estudo das Águas Subterrâneas do Calcáreo Bambuí-Bahia - Região Central da Bahia”, dissertação de Mestrado, 75 pp, (1978); A. F. Siqueira: "Uso dos Dados Isotópicos e Químicos como Indicadores de Origem das Águas e dos Sais Dissolvidos no Aqüífero Calcário Bambuí, Irecê-Bahia”, dissertação de Mestrado, 86 pp, (1978); F. V. M. Gomes e F. C. F. Cabral: "Uso dos Isótopos Naturais do Urânio no Estudo das Águas Subterrâneas do Aqüífero Calcário Bambuí (Ba)". In: Rev. Bras. Geoc. Brasil, 11, 3, pp. 179-184, (1981).

[19] H. Carvalho: "Um Aparelho para Medir Termoluminescência e Aplicações ao Estudo de um Monocristal de Quartzo de Goiás”, dissertação de Mestrado, 46 pp, (1972); D. Nordemann, e T. Sakai: "A Radioatividade do Meteorito de Marília (Queda no dia 5/outubro/71) Medida por Espectrometria Gama”, In: Rev. Bras. Geoc., Brasil, 3, 1, pp. 48-52, (1973); G. P. Shignolfi, and T. Sakai: "Uranium and Thorium in Potash Rich Rhyolites from Western Bahia (Brazil)":. In: Chem. Geol., Holanda, 14, 1, pp. 23-30, (1974); M. M. F. D'Aguiar Neto: "Estudo de Defeitos em Quartzo Enfumaçado da Bahia por Difração de Raios X”, dissertação de Mestrado, 75 pp, (1974); A. M. R. Azevedo:“Concentração e Distribuição dos Elementos Radioativos Naturais em Solos Tropicais e SemiÁridos da Fazenda Bela Vista-Bahia”, dissertação de Mestrado, 57 pp, (1975); Y. H. Pregnolatto: "Estudo de Alguns Organismos Carbonatados Marinhos Atuais, Através da razão das Atividades U234/U238 e o Teor de Urânio,
Utilizando a Técnica de Espectrometria Alfa”, dissertação de Mestrado, 73 pp, (1975); C. Ferreira: "Espectrometria Gama de Campo - Determinação das Condições de Detecção, Aplicação ao Mapeamento de Uma Jazida de Apatita", dissertação de Mestrado, 75 pp, (1973); J. Ferreira: "Estudo da Termoluminescência de Quartzito numa Zona Mineralizada em Ouro e Urânio (Canavieiras - Jacobina - Bahia)", dissertação de Mestrado, 86 pp, (1973); J. Ferreira e D. Nordemann: "Estudo da Termoluminescência Natural Quartzito, em Uma Zona Mineralizada em Ouro e Urânio (Canavieiras, Jacobina-Bahia)", In: Rev. Bras. Geoc, Brasil, 6, 4, pp. 264-277, (1976); K. Suguio, L. Martin e J. M. Flexor: "Les Variations Relatives du Niveau Moyen de la Mer au Quartenaire Recent dans la Région de Cananéia-Iguape-São Paulo", In: Boletim do Instituto de Geociências da USP (IGUSP), São Paulo, Brasil, 7, pp. 113-129, (1976); T. Sakai, and G. P. Shignolfi: "Uranium and Thorium in Archean Facies Terrains of Bahia (Brazil)", In: Geochem. Journ. Japan, 11, 1, pp. 30-33, (1977); T. Sakai: "Distribuição de $\mathrm{Cs}^{137}$ nos Solos do Estado da Bahia", dissertação do Mestrado, 63 pp, (1977); N. M. A. C. C. Pinto: "Determinações Isotópicas do Carbono e Oxigênio em Rochas Metassedimentares do Grupo Rio Pardo-Bahia", dissertação de Mestrado, 61 pp, (1977); A. Novaes: "Contribuição ao Estudo da Taxa de Intemperização dos Minerais e Rochas na Bacia de Drenagem do Rio Paraguaçu-Bahia", dissertação de Mestrado, 86 pp, (1978); H. S. Carvalho:"Método para Determinaçào de Fluxo Geotérmico com Aplicação às Bacias Sedimentares Petrolíferas do Recôncavo Baiano (Brasil) e Sumatra (Indonésia)", tese do doutorado, 92 pp, (1981) e E. J. C. A. Jucá: "Um Novo Método para Construção de Filtros Recursivos Uni e Bidimensionais de Fase Nula, com Aplicação a Campos Potenciais", tese de doutorado, 164 pp, (1981).

[20] A. Ribeiro Filho, Memória do IFUFBA - 1963 / 1984. IF. UFBA. Vol. 1, 178 pp. Salvador - Bahia (1985).

[21] A partir de 1984 foi desenvolvido o projeto "Memória", coordenado por A. Ribeiro Filho, no qual foram editados os Cadernos do IFUFBA (oito números); A. Ribeiro Filho, O. Freire Jr e J. A de L Rocha: "Modernização do Ensino da Matemática e Física no Estado da Bahia”, In: Anais do I Seminário Nacional de História da Matemática (SNHM). Recife. Pe. Sociedade Brasileira de História da Ciência (SBHC), pp. 59-63, (1998). Para a fundação do IMF, ver também A. L. M. Dias: "Engenheiros, mulheres, matemáticos: interesses e disputas na profissionalização da Matemática na Bahia (1896-1968) ". Tese de Doutoramento apresentada à FFLCH, USP (2002).

[22] J. Leite Lopes: “Ciência e Libertação". Edit. Paz e Terra, p. 145 (1969).

[23] S. Schwartzman, op.cit., p. 286.

[24] A. da S. Ramos: "Depoimento". Cadernos do IFUFBA, ano 1, no 2, pp. 31-42, maio (1985).

[25] A. da S. Ramos, op.cit., p. 40. 